\title{
Effects of search mode and intertrial priming on singleton search
}

\author{
DOMINIQUE LAMY and TOMER CARMEL \\ Tel Aviv University, Tel Aviv, Israel \\ HOWARD E. EGETH \\ Johns Hopkins University, Baltimore, Maryland \\ and \\ ANDREW B. LEBER \\ Yale University, New Haven, Connecticut
}

\begin{abstract}
There is no consensus as to what information guides search for a singleton target. Does the most salient display element capture attention, regardless of the observer's attentional set? Do observers adopt a default salience-based search mode? Does knowledge of the target's defining featural property (when available) affect search? Finally, can intertrial contingencies account for the disparate results in the literature? We investigated search for a shape singleton when (1) the target and nontarget shapes switched unpredictably from trial to trial, (2) the target feature remained fixed, and (3) the target was a singleton on only one third of the trials. We examined overall reaction times, search slopes, errors, and the magnitude of the slowing caused by a cross-dimensional singleton distractor. Our results argue against the idea that search is guided solely by stimulus-driven factors or that subjects adopt a singleton detection mode that is blind to feature information. They show also that intertrial contingencies, although potent, cannot account for the variety of results in the literature.
\end{abstract}

Attention refers to the mechanisms involved in the selection of those portions of the available sensory information that will receive processing priority. Two sources of control over attentional selection have been distinguished. Attention may be captured by a salient visual event (stimulusdriven, or bottom-up, control of attention), or attention may be directed to objects that possess task-relevant properties (goal-directed, or top-down, control of attention). There has been considerable debate over how these factors interact to determine processing priority (for recent reviews, see Rauschenberger, 2003; Ruz \& Lupiáñez, 2002).

Theeuwes (e.g., 1992) proposed that in parallel search, attention is automatically directed to the most salient object. He showed that when observers are engaged in parallel search for a particular singleton (e.g., a unique circle among diamonds), an irrelevant singleton that is more salient than the target singleton (e.g., a red element among green ones) interferes with search, even though the observers know that they have to ignore the irrelevant singleton. On the basis of such findings, Theeuwes (1992) concluded that parallel search ${ }^{1}$ is driven exclusively by

Support for this research was provided by Binational Science Foundation Grant 200267 to D.L. and H.E.E. and by Israel Science Foundation Grant 1382-04 to D.L. Correspondence concerning this article should be addressed to D. Lamy, Department of Psychology, Tel Aviv University, Ramat-Aviv, P.O. Box 39040, Tel Aviv 69978, Israel (e-mail: domi@ post.tau.ac.il). bottom-up factors, because knowledge of the target feature failed to override bottom-up interference from the most salient item in the display.

Bacon and Egeth (1994) questioned this conclusion. Using a distinction initially suggested by Pashler (1988), they proposed that in Theeuwes's (1992) experiments, two search strategies were available: (1) the singleton detection mode, in which attention is directed to the location with the largest local feature contrast, and (2) the feature search mode, which entails directing attention to items possessing a specific visual feature. Indeed, in Theeuwes's (1992) experiments, the target was defined as being a singleton and as possessing the target attribute. If subjects used the singleton detection mode, both relevant and irrelevant singletons could capture attention, depending on which exhibited the greatest local feature contrast. Bacon and Egeth first conducted a replication of Theeuwes's (1992) study. They presented subjects with displays consisting of colored circles or diamonds arranged on the circumference of an imaginary circle. Line segments varying in orientation appeared inside each item. The subjects were required to determine the orientation of the line segment within a target item. The target item was defined as the unique green diamond among green circles. As was expected, time to find the target shape singleton increased when an irrelevant color singleton (a red circle) was also present. Bacon and Egeth then introduced two manipulations designed to render the singleton detection mode inappropriate for performing the task. They presented either 
up to three identical target shapes on each trial (Experiment 2) or up to two different unique shapes in addition to the unique target shape (Experiment 3), so that the target was the unique shape singleton on only one third of the trials. These manipulations ensured that the target could not be found by simply looking for a singleton. As a result, the disruption caused by the unique distractor disappeared, even on the minority of trials in which the target was the unique shape singleton. They concluded that irrelevant singletons may or may not cause distraction during parallel search for a known singleton target, depending on the search strategy employed.

Bacon and Egeth's (1994) findings are important because they refute the idea that capture by irrelevant singletons in parallel search results from purely bottom-up processes (e.g., Theeuwes, 2004) and suggest, instead, that attentional priority depends on task requirements (see Folk, Remington, \& Johnston, 1992, for a similar view). Although the distinction between the singleton detection and the feature search modes provides a reasonable explanation for Bacon and Egeth's results and for various other findings in the literature (e.g., Folk, Leber, \& Egeth, 2002; Lamy \& Egeth, 2003; Lamy, Leber, \& Egeth, 2004; Lamy \& Tsal, 1999; Lamy, Tsal, \& Egeth, 2003; Leber \& Egeth, 2003, 2006; Ruz \& Lupiáñez, 2002; Yantis \& Egeth, 1999; but see Theeuwes, 2004), several of its aspects remain open for further enquiry.

First, the singleton detection mode is assumed to be a default search mode. Indeed, subjects are assumed to use the singleton detection mode when the feature search mode is also available, although the latter is more attractive from a probabilistic viewpoint than is the singleton detection mode: It would guide attention directly to the target on $100 \%$ of the trials, whereas the singleton detection mode would direct attention to the wrong singleton on $50 \%$ of the trials. What might explain this behavior? Bacon and Egeth (1994) suggested that the singleton detection mode may be cognitively less demanding. This might imply that the singleton detection mode leads subjects to the target more quickly than does the feature search mode. However, reaction times (RTs) in the no-distractor condition did not actually appear to be shorter in Bacon and Egeth's Experiment 1 , in which the subjects were assumed to have used the singleton detection mode, than in their Experiments 2 and 3 , in which they were assumed to have used the feature search mode. But because the different conditions were run on different subjects in different experiments, such comparisons may not be sufficiently informative.

Second, a strong interpretation of the notion of a default singleton detection mode implies that when the target shape is known and is always a unique singleton (i.e., when singleton-ness is also a defining feature of the target), attention is directed to the most salient item in the display (singleton detection mode), regardless of whether or not this item possesses the target shape. The same mechanism is thus assumed to underlie search for a known singleton and search for an unknown singleton.

Findings presented by Bravo and Nakayama (1992) argue against the latter assumption and suggest that search for a known singleton is different from search for an unknown singleton. These authors (see also Egeth, Jonides, \& Wall, 1972) compared subjects' performance on two different conditions of search for a pop-out target (e.g., a single red target among green nontargets). In the blocked condition, the target and nontarget colors were known and did not vary throughout a whole block of trials. In the mixed condition, the target and nontarget colors were switched unpredictably from trial to trial. The pattern of results in the two conditions differed on two notable aspects. First, RTs were substantially shorter (by about $100 \mathrm{msec}$ ) in the blocked than in the mixed condition. Second, whereas in the blocked condition RTs remained constant as the number of nontargets increased, a negative slope was observed in the mixed condition.

Bravo and Nakayama (1992) concluded that the mechanism that one uses to perform a pop-out search task depends on whether or not the target feature is known. Specifically, in the blocked condition, subjects use a feature-based strategy in which attention is guided by knowledge of the target feature. In the mixed condition, in which the target feature switches unpredictably from trial to trial, subjects use a salience-based strategy in which attention is directed to the most salient object in the visual field.

To summarize, Bacon and Egeth (1994) suggested that search for a known singleton is not guided by knowledge of the target feature but by knowledge that the target is a singleton and based this conclusion on the differential effects of an irrelevant singleton on search performance in the singleton detection (Experiment 1) versus feature search (Experiments 2 and 3 ) conditions. Bravo and Nakayama (1992) reached the opposite conclusion on the basis of differences in RTs and slopes between the mixed and the fixed conditions: They proposed that search for a known singleton is not guided by stimulus-driven salience but exclusively by knowledge of the target feature. A series of experiments conducted by Maljkovic and Nakayama (1994) seems to resolve this apparent contradiction.

Maljkovic and Nakayama (1994) overturned Bravo and Nakayama's (1992) conclusion by proposing that the dramatic RT advantage observed in the blocked condition, relative to the mixed condition, does not result from explicit knowledge of the target feature but from intertrial repetition effects. In a series of experiments involving several variants of Bravo and Nakayama's mixed condition, Maljkovic and Nakayama (1994) showed that performance is enhanced if the target has the same feature on consecutive trials. They found such repetition effects, which they called "priming of pop-out" (henceforth, PoP), to cumulate over up to eight consecutive same target feature trials, at which point performance reaches the RT level observed in the blocked condition. On the basis of this new set of data, these authors concluded that the same salience-based mechanism underlies pop-out search, whether or not the target feature is known. ${ }^{2}$ This conclusion converges with Bacon and Egeth's assumption that subjects use the singleton detection mode and do not exploit explicit knowledge of the target feature when this is available, as long as the target is always a singleton. 
The PoP phenomenon has proved to be a robust phenomenon (e.g., Goolsby \& Suzuki, 2001; Hillstrom, 2000; Hodsoll \& Humphreys, 2001; Wolfe, Butcher, Lee, \& Hyle, 2003). In contrast, there is weaker support for Maljkovic and Nakayama's (1994) claim that the mechanism underlying search is the same whether the target feature is known or unknown and that the shorter RTs observed in the former type of search can be entirely accounted for by PoP effects. Three considerations lead us to question such a claim.

First, in an experiment modeled after Maljkovic and Nakayama's (1994), Hillstrom (2000, Experiment 2) observed that repetition priming of a moderately salient target-defining feature, on the orientation dimension, did not cumulate over several consecutive trials. That is, multiple consecutive repetitions had no stronger effect than did a single repetition, which decreased mean RTs from approximately 1,150 to $1,050 \mathrm{msec}$. However, RTs on such repeated target feature trials in the mixed condition remained strikingly longer than RTs in the blocked condition (1,050 vs. $720 \mathrm{msec}$, respectively). Thus, there remained an RT advantage when the target feature was known, relative to when it was unknown, that was not eliminated by PoP.

Second, closer scrutiny of the findings presented by Maljkovic and Nakayama (1994) suggests that they might result from an artifact in the procedure used. In their Experiment 7, these authors showed that when cumulative priming reached its asymptote, RTs in a variant of the mixed condition did not differ from RTs in the baseline, blocked condition. However, in the mixed condition used in Maljkovic and Nakayama's (1994) experiment, the probability of a target feature change on the next trial was much lower than the probability that the target feature would remain the same. Indeed, subjects were run on sequences of same-color trials of variable lengths $(2$, 6 , and 10 for one subject and 4, 8, and 11 for the other subject). It follows that, on average for the first subject, for instance, there was a probability of $3 / 18$ for a target feature change and of 15/18 for a same-color target on the next trial. Thus, it is possible that expectancy effects affected performance, in addition to PoP effects. In that case, cumulative repetition effects would not account for all the difference between the target feature known and the target feature unknown conditions. ${ }^{3}$

Our third consideration is that assuming that PoP can account for the RT difference between the blocked and the mixed conditions despite the foregoing reservations, it is not clear how PoP also accounts for the difference in search slopes between the two conditions. Remember that Bravo and Nakayama (1992) had observed that RTs decreased as the number of nontargets (and therefore, display density) increased in the mixed condition, whereas RTs remained constant in the blocked condition (for replications of this finding, see Maljkovic \& Nakayama, 1994, Experiment 1; Wolfe et al., 2003). This observation fits nicely with Bravo and Nakayama's initial account, according to which search in the mixed condition was driven by local contrast and, therefore, improved with increased display density, whereas search in the blocked condition was guided by top-down knowledge of the target feature and was, thus, insensitive to density effects. But why this difference in slopes if search was salience-based in both conditions, as Bacon and Egeth (1994) and Maljkovic and Nakayama (1994) claimed? One would have to assume that PoP interacts with display size - namely, that density effects wane with more repetitions. Because the studies investigating PoP either did not involve display size manipulations (e.g., Hillstrom, 2000; Maljkovic \& Nakayama, 1994) or did not report repetition effects as a function of display size (e.g., Wolfe et al., 2003), it is not known at this point whether there is, indeed, such an interaction.

The objective of the present study was to test the notion of a default singleton detection mode that is blind to additional feature information - that is, in operational terms, to test the idea that the same mechanism (the singleton detection mode) underlies pop-out search when the target feature is known (blocked condition; henceforth, the fixed singleton condition) and when it is unknown (mixed condition; henceforth, the mixed singleton condition) and that this mechanism is faster than search guided by the target's known feature (the feature search mode).

\section{EXPERIMENT 1}

We compared the three different types of search studied by Bacon and Egeth (1994) and Maljkovic and Nakayama (1994) in a within-subjects design. Our experimental strategy was similar to that of these authors, in that we manipulated the search strategies available for finding the target but compared performance on exactly the same displays in each condition.

In the mixed singleton condition, the target and nontarget shapes were randomly switched across trials, as in Bravo and Nakayama's (1992) mixed condition. In the fixed singleton condition, the target and nontarget shapes remained constant throughout the block, as in Bravo and Nakayama's fixed condition and in Bacon and Egeth's (1994, Experiment 1) replication of Theeuwes's (1992) experiment. Finally, in the multiple target condition, there were one, two, or three identical targets on each trial, defined by their known shape, as in Bacon and Egeth's Experiment 2. Displays consisted of colored circles or diamonds arranged on the circumference of an imaginary circle. A line segment (either horizontal or vertical) appeared inside each item. The subjects were required to determine the orientation of the line segment within a target item. Display size was either five or nine. All the items in the displays had the same color on distractor-absent trials ( $50 \%$ of the trials), and one nontarget was a color singleton on distractor-present trials ( $50 \%$ of the trials).

We expected to replicate previous findings. In line with Bravo and Nakayama's (1992) findings, we expected higher RTs in the mixed condition than in the fixed singleton condition and negative slopes as a function of display size in the mixed singleton condition versus flat slopes in the fixed singleton condition. We also expected to replicate the PoP effects observed by Maljkovic and Nakayama 
(1994) — that is, shorter RTs on repeated versus switched target feature trials in the mixed singleton condition. Finally, in line with Bacon and Egeth's (1994) findings, we expected higher RTs in the distractor-present than in the distractor-absent condition in the fixed and mixed singleton conditions but no distractor interference in the multiple target condition.

In addition, we tested a number of novel predictions that follow from the proposal that subjects adopt a default singleton detection search mode when the target is a known singleton. First, if searching for the most salient element is intrinsically faster than searching for a known feature, RTs should be shorter in the fixed singleton condition than on one-target trials in the multiple target condition. Second, if $\mathrm{PoP}$ is to account for all the differences between the fixed and the mixed singleton conditions, RTs, search slopes, and the magnitude of distractor interference in the two search conditions should become similar on repeated feature trials.

\section{Method}

\section{Subjects}

The subjects were twenty-four Tel Aviv University undergraduate students who participated in the experiment for course credit. All reported having normal or corrected-to-normal visual acuity and normal color vision.

\section{Apparatus}

Displays were generated by an Intel Pentium 4 computer attached to a 15 -in. TFT monitor, using $640 \times 480$ resolution graphics mode. Responses were collected via the computer keyboard. A chinrest was used to set viewing distance at $50 \mathrm{~cm}$ from the monitor.

\section{Stimuli}

Examples of the stimulus displays are presented in Figure 1. The fixation display was a gray $0.2^{\circ} \times 0.2^{\circ}$ plus sign $(+)$ in the center of a black background. Stimulus displays consisted of the fixation display with the addition of five or nine colored shapes, circles, or diamonds, presented equally spaced along the circumference of an imaginary circle, centered at fixation. At a viewing distance of $50 \mathrm{~cm}$, the centers of the shapes were $3.4^{\circ}$ from fixation. Display density thus increased with number of shapes. Circles subtended $1.4^{\circ}$ in diameter, and diamonds were $45^{\circ}$-rotated squares, $1.6^{\circ}$ on a side. Centered inside each shape was a white horizontal or vertical line segment $\left(0.5^{\circ}\right.$ in length). There were three search conditions. In the fixed singleton condition, the target was a circle, and the nontargets were diamonds on each trial for half the subjects; for the other half of the subjects, the target was a diamond, and the nontargets were circles. Displays were identical in the mixed singleton condition, except that for each subject, the target and the nontarget shapes were switched unpredictably from trial to trial, instead of remaining constant. Displays in the multiple target condition were identical to those in the fixed singleton condition, except that rather than only one target appearing on each trial, up to three targets (each containing a line segment of the same orientation) appeared. In the distractorabsent condition, all the shapes in the display were presented in the same color. In the distractor-present condition, one of the nontargets was presented in a different color. For half of the subjects, the color singleton distractor was red (CIE coordinates, .630/.340), and the other shapes were green (CIE coordinates, .280/.591). This color assignment was reversed for the other half of the subjects. The red and green colors were matched for luminance $\left(21\right.$ and $20.5 \mathrm{~cd} / \mathrm{m}^{2}$, respectively), using a light meter (Minolta ColorCAL colorimeter). All the stimuli were drawn with a 2-pixel stroke.

\section{Procedure}

The subjects had to determine the orientation of the line segment inside the target shape and to respond by pressing designated keys on the computer keyboard (the " $z$ " key for horizontal and the " 3 " keypad key for vertical) as quickly as possible, while maintaining high accuracy. Error trials were followed by a 500 -msec feedback beep. In the mixed singleton condition, the subjects were told that the target was the shape with the unique form - that is, the unique circle among diamonds on some trials and the unique diamond

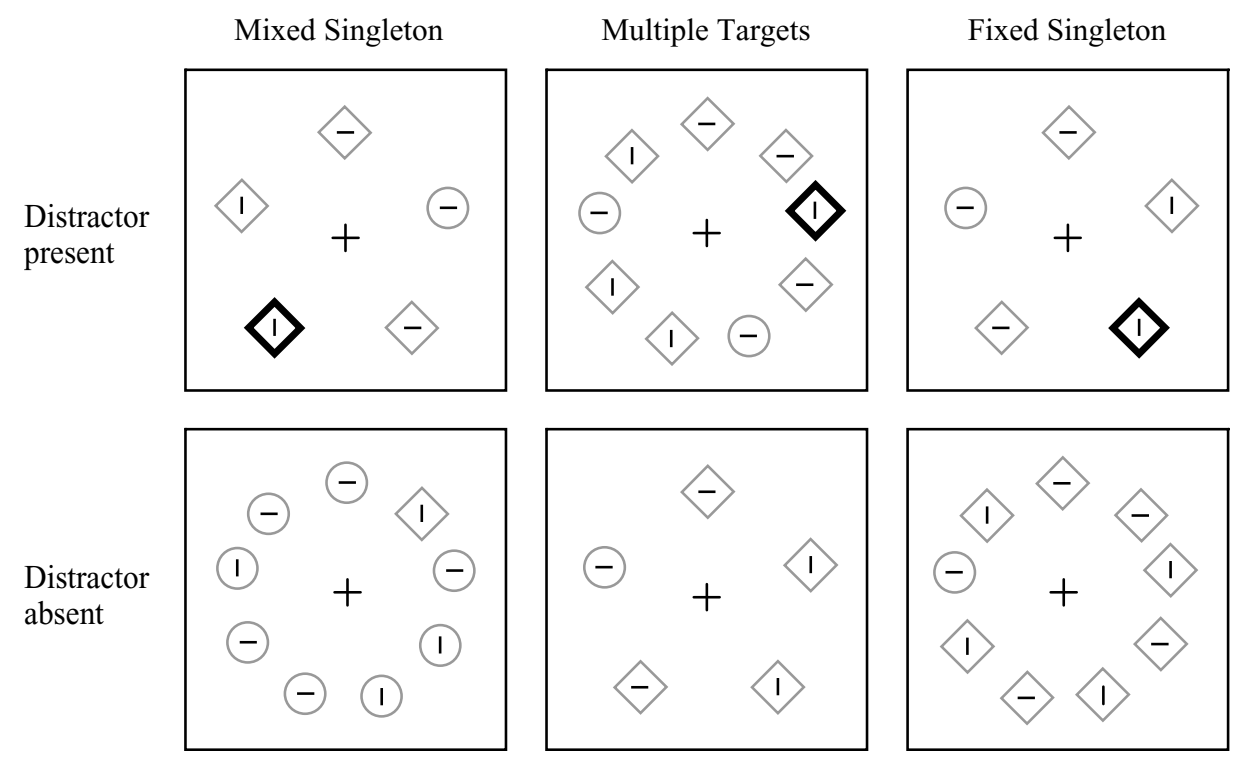

Figure 1. Examples of stimulus displays for each condition of search type and distractor presence. The thick black stroke corresponds to the color of the irrelevant singleton (red or green), and the thin gray stroke designates the color of the remaining elements (green or red, respectively). The thin black stroke was gray. 
among circles on other trials. In the fixed singleton condition, the subjects were told that the target shape was always the diamond (or the circle). The same instruction was given in the multiple target condition, except that the subjects were told that more than one target could be present and that, in this case, the lines within each target shape would always be the same. For any given subject, the same target shape was assigned for the fixed singleton and the multiple target conditions.

Each trial began with the presentation of the fixation display. After $350 \mathrm{msec}$, it was replaced by the stimulus display, which remained visible for $2,000 \mathrm{msec}$ or until response. The screen went blank for $500 \mathrm{msec}$ before the next trial began. Eye movements were not monitored, but the subjects were explicitly requested to maintain fixation throughout each trial.

\section{Design}

There were three between-subjects variables: order of search condition presentation (six possible orders), target shape in the fixed singleton and multiple target conditions (circle or diamond), and distractor color (red or green). For balance, each of the 24 subjects was randomly assigned to one of the 24 possible between-subjects conditions. There were three within-subjects variables: search condition (mixed singleton, fixed singleton, or multiple target), distractor presence (present or absent), and display size (five or nine). Search conditions were presented in separate blocks of trials. Conditions of distractor presence and display size were randomly intermixed within each block of search condition. In the mixed singleton condition, the target was equally likely to be a diamond or a circle. The target shape was equally likely to appear in any of the five or nine possible locations. The distractor, when present ( $50 \%$ of the trials), was equally likely to appear in the remaining four or eight possible locations. The line segment inside the target shape was equally likely to be vertical or horizontal. On each trial, line orientation was randomly assigned to each shape in the display, with the constraints that the number of lines of each orientation in each display always differed exactly by one and that all the targets within a display contained the same line orientation (multiple target condition).

Each search condition began with one block of 50 practice trials, followed by one block of 240 trials. Thus, the experiment included 150 practice trials and 720 experimental trials. The subjects were allowed a rest period after each block.

\section{Results}

To preview, the main findings by Bacon and Egeth (1994) and Maljkovic and Nakayama (1994) were closely replicated. However, the new tests used in the present experiment did not generally support the idea of a default singleton detection mode.

Figure 2 shows mean RTs on correct trials and the accuracy data for Experiment 1. Note that the data are for identical displays - that is, displays with one target - and the multiple target data for two- and three-target trials are, therefore, not shown. In all the RT analyses, error trials (6.6\% of all the trials) were removed from analysis, and RTs for each subject were sorted into cells by search condition, distractor presence, and display size. RTs exceeding the mean of a given cell by more than 3.5 standard deviations were trimmed. This removed fewer than $1 \%$ of all the observations. ${ }^{4}$

\section{Overall Analysis}

An ANOVA was conducted with search condition (mixed singleton, fixed singleton, or multiple target), distractor presence (present or absent), and display size (five or nine) as within-subjects factors. Only trials with identical displays in the three conditions were entered in this
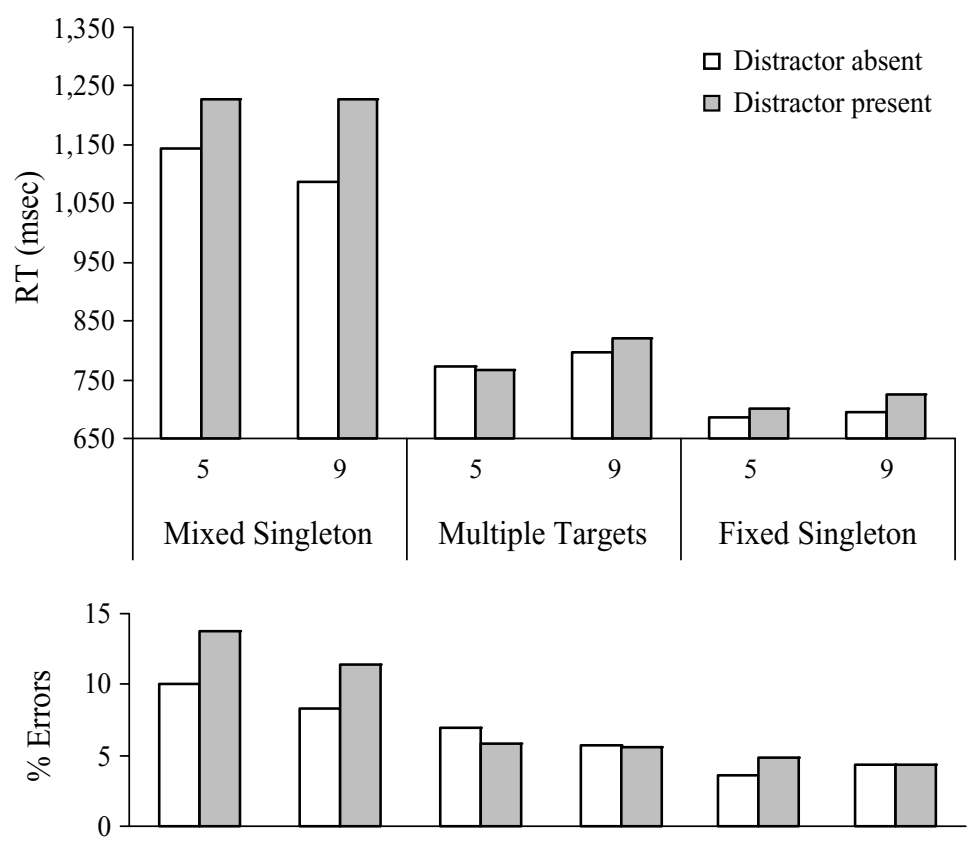

Figure 2. Experiment 1: Target identification performance by conditions of search type (mixed singleton, fixed singleton, or multiple target), display size, and distractor presence. Upper panel: Mean reaction times (RTs) in milliseconds. Lower panel: Percent errors. 
analysis - that is, for the multiple target condition, only one-target trials were included.

Reaction times. Main effects of search condition and distractor presence were significant $[F(2,44)=205.26$, $p<.0001$, and $F(1,22)=39.19, p<.0001$, respectively $]$. The main effect of display size was nonsignificant $(F<1)$. The significant main effects were modulated by the following interactions.

The interaction between search condition and distractor presence was significant $[F(2,44)=19.73, p<.0001]$. Paired comparisons showed a significant distractor interference in the mixed singleton condition $[112 \mathrm{msec} F(1,22)=$ $38.56, p<.0001]$ and in the fixed singleton condition [23 msec; $F(1,22)=12.67, p<.002]$, with a significantly larger effect in the former condition $[F(1,22)=27.85, p<$ $.0001]$. There was no distractor interference in the multiple target condition ( $9 \mathrm{msec} ; F<1$ ). Because we were interested in comparing response speeds in the three search conditions and the presence of the distractor had differential effects in each condition, it was important to compare RTs in the distractor-absent condition: Responses in the mixed singleton condition were slower than those in the fixed singleton condition by $329 \mathrm{msec}[F(1,22)=301.95$, $p<.0001]$, and responses in the multiple target condition were slower than those in the fixed singleton condition by $96 \operatorname{msec}[F(1,22)=31.12, p<.0001]$.

There was a significant interaction between display size and distractor presence $[F(1,22)=13.38, p<.002]$. This effect did not interact with search conditions $(F<1)$, indicating that distractor interference increased with display density across search conditions: 81 versus $143 \mathrm{msec}$ in the mixed singleton condition, 14 versus $30 \mathrm{msec}$ in the fixed singleton condition, and -8 versus $23 \mathrm{msec}$ in the multiple target condition.

Finally, the interaction between search condition and display size was significant $[F(2,44)=9.47, p<.0004]$. Because distractor presence interacted with display size, it was more appropriate to compare search slopes in each search condition with no distractor: Paired comparisons revealed a significant difference in slopes between the fixed and the mixed singleton conditions $[F(1,22)=9.71$, $p=.005]$ and no difference between the fixed singleton and the multiple target conditions $(F<1)$. Search slopes were significantly negative in the mixed singleton condition $[-14$ msec per item; $F(1,22)=14.49, p<.001]$ and did not significantly differ from zero in the multiple target and fixed singleton conditions $(6.25$ and $1.75 \mathrm{msec} / \mathrm{item}$, respectively; $F \mathrm{~s}<1)$.

Accuracy. Main effects of search condition and distractor presence were significant $[F(2,44)=24.84$, $p<.0001$, and $F(1,22)=6.54, p<.02$, respectively]. The interaction between these factors was significant $[F(2,44)=5.36, p<.009]$. Paired comparisons showed that the subjects made more errors on distractor-present than on distractor-absent trials in the mixed singleton condition $[13 \%$ vs. $9 \%$, respectively; $F(1,22)=14.49, p<$ $.003]$, but no such difference was observed in either the fixed singleton or the multiple target condition $\left(F_{\mathrm{S}}<1\right)$. On distractor-absent trials, the subjects made more errors in the mixed than in the fixed singleton condition $[9 \%$ vs. $4 \%$, respectively; $F(1,22)=30.41, p<.0001]$ and in the multiple target condition than in the fixed singleton condition $[6 \%$ vs. $4 \%$, respectively; $F(1,22)=7.52, p<$ $.02]$. All the significant effects on the accuracy data thus mirrored those observed on the RT data, so that a speedaccuracy trade-off was not a concern.

\section{Target Feature Repetition Effects ${ }^{5}$}

The objective of the following analyses was to determine whether the differences observed between the mixed and the fixed singleton conditions would be eliminated or at least reduced on repeated target feature trials (PoP), as should follow from Maljkovic and Nakayama's (1994) account. Accordingly, in the mixed singleton condition, we would expect RTs to diminish, slopes to become more positive, and distractor interference to be reduced when the target feature repeats.

To assess PoP effects in the mixed singleton condition, we conducted an ANOVA with target feature repetition (repetition or no repetition), display size, and distractor presence as factors. Trials with one or more target feature repetitions were included within the same level because there was an insufficient number of trials to analyze the effect of more than one repetition separately. Figure 3 presents target feature repetition effects for different conditions of distractor presence and display size.

Reaction times. The main effect of target feature repetition was significant $[F(1,22)=72.53, p<.0001]$, with shorter RTs on repeated target feature trials (1,108 vs. $1,200 \mathrm{msec})$. This effect interacted with neither display size $[F(1,22)=2.22, p>.1]$ nor distractor interference $(F<1)$.

Accuracy. The main effect of target feature repetition was significant $[F(1,22)=17.50, p<.0004]$, with fewer errors on repeated target feature trials. No interaction involving this factor approached significance $($ all $F \mathrm{~S}<1)$.

\section{Discussion}

As was expected, previous findings were replicated: (1) long RTs and a negative search slope in the mixed singleton condition versus short RTs and a flat search slope in the fixed singleton condition (Bravo \& Nakayama, 1992); (2) priming of pop-out effects (i.e., shorter RTs on repeated than on switched target feature trials in the mixed singleton condition [PoP; Maljkovic \& Nakayama, 1994]); and (3) distractor interference in the fixed singleton condition, not in the multiple target condition (Bacon \& Egeth, 1994). Note, however, that distractor interference increased with display size across the different types of search, suggesting a role for stimulus-driven salience irrespective of task demands. Specifically, in the multiple target condition, distractor interference grew from $9 \mathrm{msec}$ in five-item displays to $23 \mathrm{msec}$ in nine-item displays (see Lamy, Bar-Anan, Egeth, \& Carmel, 2006, for a similar finding). Although the latter effect was nonsignificant $[F(1,22)=1.62, p>$ $.2]$, it is possible that it may reach significance with even larger set sizes (e.g., Theeuwes, 2004). 


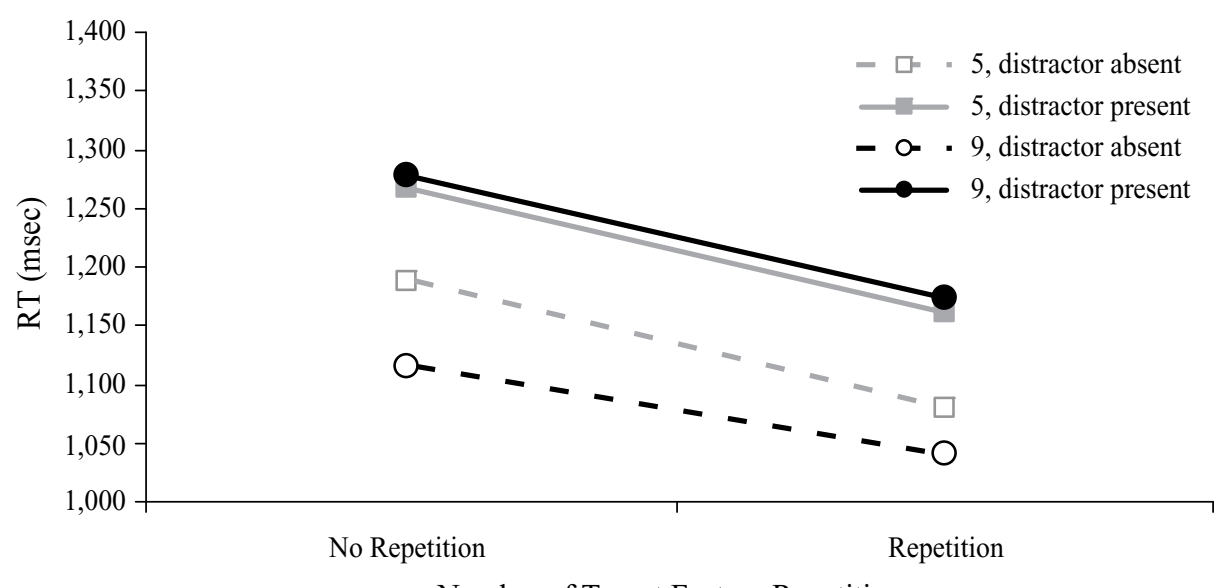

Number of Target Feature Repetitions

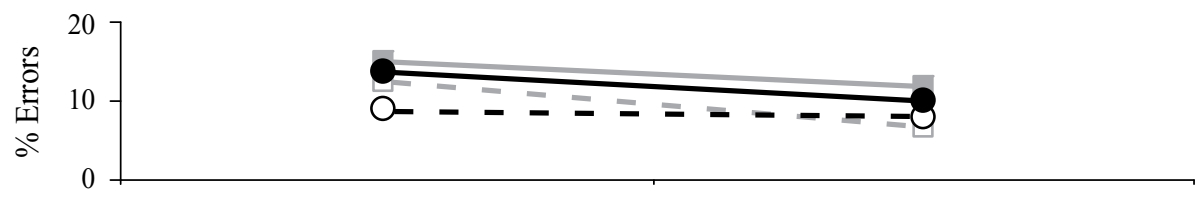

Figure 3. Experiment 1: Target identification performance on trials in which the target and distractor features were the same versus differed in the preceding trial, for each condition of search type, display size, and distractor presence. Upper panel: Mean reaction times (RTs) in milliseconds. Lower panel: Percent errors.

We obtained mixed results as to the existence of a default singleton detection mechanism underlying pop-out search. On the one hand, for identical displays (one-target trials), the subjects were faster when the target was always a singleton (fixed singleton condition) than when this happened only on one third of the trials (multiple target condition), which suggests that to the extent that the subjects used a salience-based mode in the fixed singleton condition, this mode was, indeed, faster than the feature search mode. On the other hand, the mixed and fixed singleton conditions differed in mean RTs, magnitude of distractor interference, and search slopes. Although PoP reduced RT differences, it did not modulate differences in either vulnerability to distractor interference or search slopes between the two conditions.

\section{EXPERIMENT 2}

In Experiment 1, performance in the mixed and fixed singleton conditions presented several differences (overall RTs, search slopes, and magnitude of distractor interference), ${ }^{6}$ and among these, only the overall difference in RTs was reduced by target feature repetition (PoP). However, our experimental conditions allowed us to examine the effects of only one target feature repetition. It was thus important to compare the fixed and the mixed singleton conditions using longer runs of target feature repetitions (up to three repetitions). This was the objective of Experiment 2. The extra trials needed to achieve longer runs of target feature repetition were obtained by eliminating the multiple target condition.
Note that Maljkovic and Nakayama (1994) observed that PoP effects reached their asymptote after about seven repetitions. However, as was explained in the introduction, in order to achieve such long target feature repetition runs, these authors had their subjects run on sequences of samefeature trials of variable lengths, so that the probability of a target feature change on the next trial was much lower than the probability that the target feature would remain the same. With the constraint that these probabilities be identical and in the context of a multifactor experiment, it was not possible to build up enough repetitions in an hour session. We thus examined PoP effects only up to three repetitions. Moreover, we anticipated that PoP effects would reach their asymptote faster in the present experiment than in Maljkovic and Nakayama's (1994), on the basis of the fact that Hillstrom (2000) found PoP for a singleton defined by its unique orientation to asymptote after just one repetition and attributed this shorter cumulative effect to the lower salience of the orientation singleton, relative to the color singleton. It was reasonable to assume that the shape singletons we used here were less salient than Maljkovic and Nakayama's (1994) color singletons.

Maljkovic and Nakayama (1994) showed that both target and nontarget feature repetition speeded search in the mixed singleton condition (Experiment 8). In the present experiment, we attempted to disentangle the effects of target and nontarget feature repetition by varying the two factors orthogonally. Accordingly, in the mixed singleton condition, the target and the nontarget shapes did not switch with one another from trial to trial but were drawn from three, rather than only two, possible shapes. We also examined the ef- 
fects of nontarget repetition in the fixed singleton condition by having the nontarget feature vary randomly between two possible values from trial to trial. Nontarget feature repetition could thus be used as an additional measure with which to compare the fixed and the mixed singleton conditions.

\section{Method}

\section{Subjects}

The subjects were 13 Tel Aviv University undergraduate students who participated in the experiment for course credit. All reported having normal or corrected-to-normal visual acuity and normal color vision.

\section{Apparatus, Stimuli, Procedure, and Design}

The apparatus, stimulus displays, and procedure were similar to those used in Experiment 1 in the mixed and fixed singleton conditions, except for the following changes. There were three possible target shapes (a circle, a diamond, or a seven-point star), instead of just two (a circle or a diamond). The seven-point star could be enclosed in a circle subtending $1.6^{\circ}$ in diameter with the points included and $1.4^{\circ}$ with the points excluded. The multiple target condition was excluded, so that there were two search conditions (fixed and mixed singleton conditions), instead of three. In the mixed singleton condition, the target had one of the three possible target shapes, and all the nontargets had one of the two remaining shapes. Target and nontarget shapes changed from trial to trial. Thus, there were six possible target-nontarget combinations in this condition. In the fixed singleton condition, the target shape remained the same throughout the block, but the nontargets switched unpredictably from trial to trial between the two remaining shapes. The subjects were randomly assigned to one of the three target shapes. Thus, for each subject, there were two possible target-nontarget combinations in this condition.

In the mixed singleton condition, there were five possible trial sequences, each of which was defined by the number of consecutive trials with the same target shape. For each of the three possible targets, there were 4 five-trial-long sequences, 4 four-trial-long sequences, 8 three-trial-long sequences, 16 two-trial-long sequences, and 32 one-trial-long sequences. This design ensured that on any given trial, there was an equal probability that the target feature on the next trial would switch or remain the same. Because this was not true for the longest (five-trial) sequence type, for which the probability of change was 1 , the corresponding trials were excluded from analysis. Thus, in contrast with the situation that prevailed in Maljkovic and Nakayama's (1994, Experiment 7) study, should repetition effects be observed in the present experiment, they would not be contaminated by top-down expectancy effects. Sequence types were randomly mixed, with the constraint that there were no consecutive sequences with the same target shape. For each sequence type, there were an equal number of distractor-present and distractor-absent trials. Nontarget shape was randomly assigned on each trial.

Each search condition began with one block of 30 practice trials, followed by one block of 372 trials. Thus, the experiment included 60 practice trials and 744 experimental trials.

\section{Results}

Despite the longer repetition runs used in the present experiment (up to three repetitions - that is, four consecutive trials with the same target feature), the dissimilarities between the mixed and the fixed singleton conditions were not diminished.

One subject was removed from the analysis due to a very high RT (more than 2.5 standard deviations above average RT). In all the RT analyses, error trials (6.7\% of all the trials) were removed from analysis, and the RTs for each subject were sorted into cells according to search condition, distractor presence, and display size. RTs exceeding the mean of a given cell by more than 3.5 standard deviations were trimmed. This removed fewer than $1 \%$ of all the observations.

\section{Overall Analysis}

Mean RTs on correct trials and the accuracy data are presented in Figure 4. An ANOVA was conducted with search condition (mixed or fixed singleton), distractor
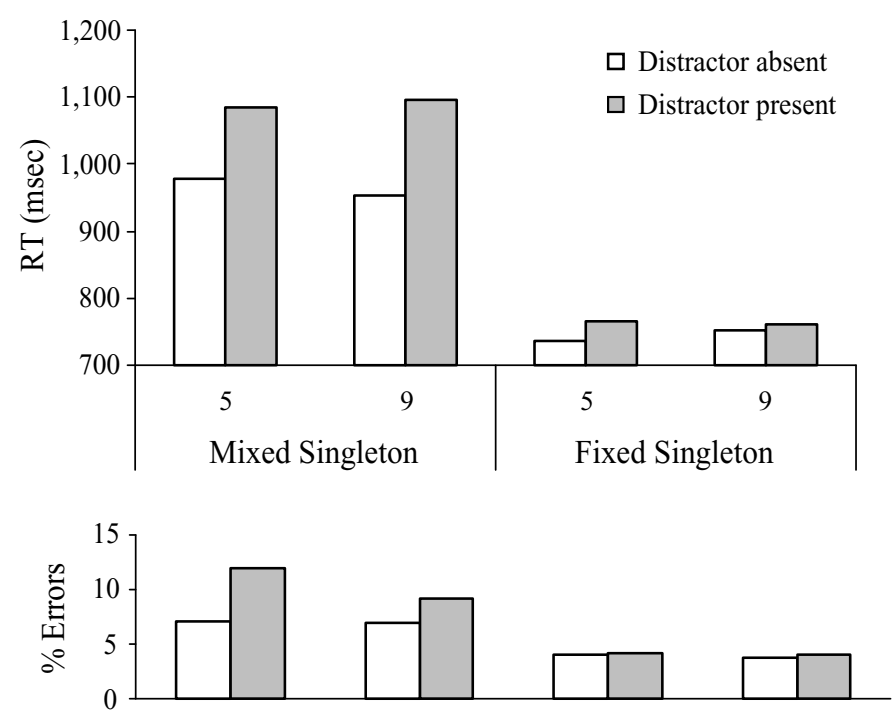

Figure 4. Experiment 2: Target identification performance by conditions of search type (mixed vs. fixed singleton), display size, and distractor presence. Upper panel: Mean reaction times (RTs) in milliseconds. Lower panel: Percent errors. 
presence (present or absent), and display size (5 or 9) as within-subjects factors.

Reaction times. The findings of Experiment 1 were replicated. The main effects of search condition and distractor presence were significant $[F(1,11)=76.34, p<$ .0001 , and $F(1,11)=86.15, p<.0001$, respectively]. The interaction between the two factors was significant $[F(1,11)=25.42, p<.0004]$, with larger distractor interference in the mixed than in the fixed singleton condition $[124 \mathrm{msec}, F(1,11)=29.19, p<.0002$, vs. $20 \mathrm{msec}$, $F(1,11)=8.34, p<.02$, respectively]. On distractorabsent trials, RTs were shorter in the fixed than in the mixed singleton condition by $222 \mathrm{msec}$. The interaction between display size and search condition was also significant $[F(1,11)=7.83, p<.02]$. On distractor-absent trials, search slopes were -13.5 msec per item $[F(1,11)=$ $5.30, p<.05]$ and $3.75 \mathrm{msec}$ per item $(F<1)$ in the mixed and the fixed singleton conditions, respectively.

Accuracy. The accuracy data mirrored the RT data. The main effects of search condition, distractor presence, and display size were significant $[F(1,11)=36.33$, $p<.0001, F(1,11)=40.79, p<.0001$, and $F(1,11)=$ $10.08, p<.009$, respectively]. The interaction between search condition and distractor presence was significant $[F(1,11)=21.45, p<.007]$. Paired comparisons revealed that there were fewer errors on distractor-absent than on distractor-present trials in the mixed singleton condition $[7 \%$ vs. $11 \% ; F(1,22)=47.40, p<.0001]$, but there was no such effect in the fixed singleton condition $(F<1)$. On distractor-absent trials, there were fewer errors in the fixed than in the mixed singleton condition $[4 \% \mathrm{vs} .7 \%$, respectively; $F(1,11)=13.28, p<.004]$.

\section{Target and Nontarget Feature Repetition Effects}

Of chief interest was whether repetition effects with longer runs in the mixed singleton condition would eliminate the RT difference between the two search conditions and would interact with distractor presence and display size so as to eliminate or at least reduce the differences between the two search conditions on distractor interference and slopes. Preliminary ANOVAs on RT and accuracy data with target feature repetition (zero, one, two, or three) and nontarget feature repetition (zero, one, two, or three) as factors showed no interaction between target and nontarget feature repetitions effects $\left(F_{\mathrm{s}}<1\right)$. Thus, in order to increase power and examine possible interactions between repetition effects and display size or distractor presence, target and nontarget feature repetition effects were examined in separate ANOVAs.

Figure 5 presents mean RTs in the mixed singleton condition as a function of number of target feature (left panel) and nontarget feature (right panel) repetitions by conditions of distractor presence and set size. RT data revealed main effects of target feature repetition $[F(3,33)=15.04$, $p<.0001]$ and of nontarget feature repetition $[F(2,22)=$ $3.17, p=.04]$. Planned comparisons showed that for both types of repetition, three consecutive repetitions had no stronger effects than two $\left(F_{\mathrm{S}}<1\right.$; see Figure 5$)$. Obtaining average RTs after both types of repetition effects had reached their asymptotes would allow us to determine whether or not, at that point, RTs in the mixed singleton condition became comparable to RTs in the fixed singleton condition. However, we could not directly measure RTs after three target feature repetitions and three nontarget feature repetitions, because there were too few trials. In order to obtain an estimate of the maximum joint contribution of target and nontarget feature repetitions to the difference between the mixed and the fixed singleton conditions, we took advantage of the fact that the two types of repetition had independent contributions (there was no significant interaction between them). We calculated the sum of the effects of three target feature repeti-
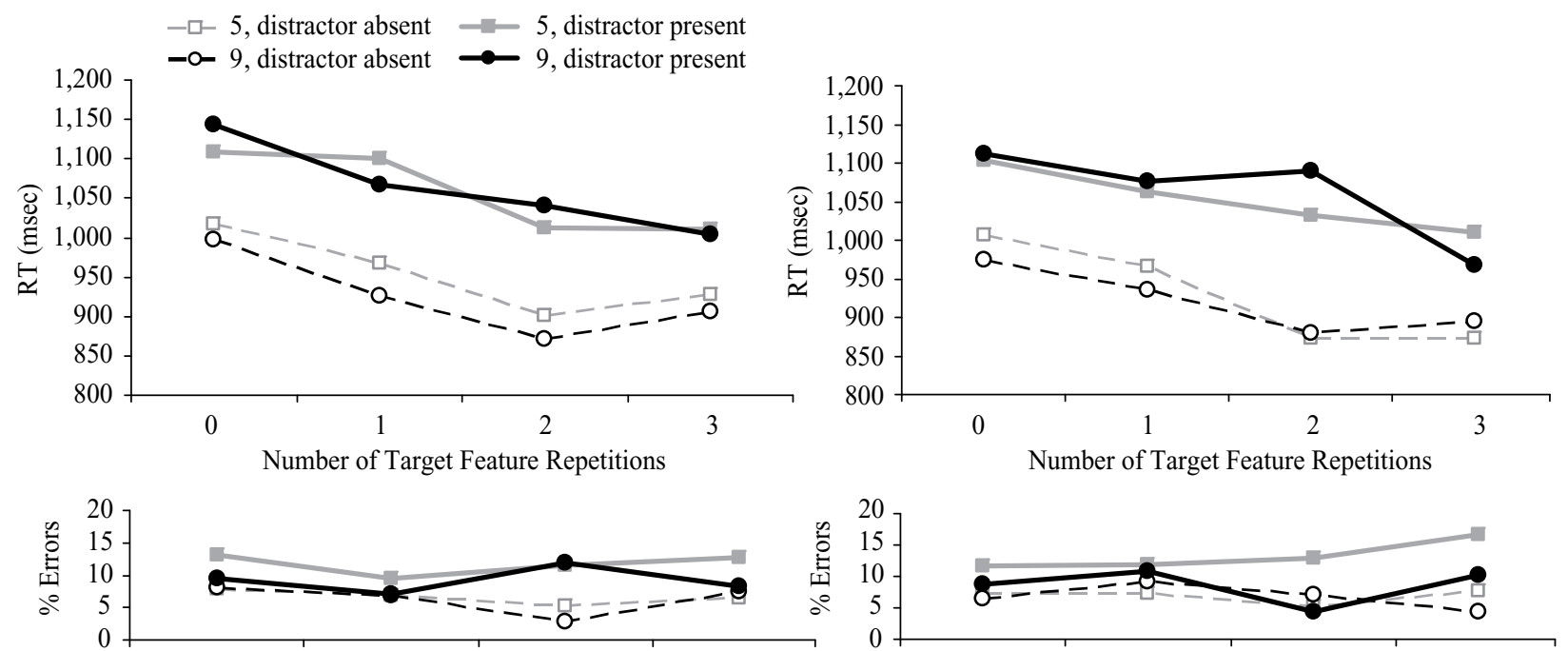

Figure 5. Experiment 2: Target identification performance as a function of the number of target feature repetitions (left panel) and the number of nontarget feature repetitions (right panel) by conditions of search type (mixed singleton vs. fixed singleton), display size, and distractor presence. Upper panel: Mean reaction times (RTs) in milliseconds. Lower panel: Percent errors. 
tions across conditions of nontarget feature repetitions (an RT reduction of $90 \mathrm{msec}$ ) and of three nontarget feature repetitions across conditions of target feature repetitions (an RT reduction of $107 \mathrm{msec}$ ) and subtracted the sum of these effects $(197 \mathrm{msec}$ ) from the average RT in the mixed singleton condition with no repetition $(1,026 \mathrm{msec})$. The estimated RT in the mixed singleton condition after the two types of effects had reached their asymptotes was thus 830 msec. It remained longer than the average RT in the fixed singleton condition ( $745 \mathrm{msec}$ ) by $85 \mathrm{msec}$.

No interaction approached significance. That is, the number of repetitions did not modulate distractor interference $[F(3,33)=2.06, p>.1$, and $F(3,33)=1.73, p>$ .1 , for target and nontarget feature repetitions, respectively]. Distractor interference was significantly larger in the mixed singleton condition after four consecutive trials with the same target feature than in the fixed singleton condition [93 vs. $20 \mathrm{msec}$, respectively; $F(1,11)=5.82$, $p<.03]$. The numerical reductions in distractor interference that can be seen in Figure 5 between the second and the third repetitions of the target feature and between the second and the third repetitions of the distractor feature were both nonsignificant $\left(F_{\mathrm{S}}<1\right)$. Moreover, the number of repetitions did not modulate the display size effect $\left(F_{\mathrm{S}}<1\right)$. Accuracy data revealed no significant effect.

Nontarget feature repetition effects were also examined for the fixed singleton condition, but no effect involving this factor approached significance.

\section{Discussion}

The results of Experiment 2 reinforce the conclusions from Experiment 1: PoP did not eliminate the differences between the fixed and the mixed singleton conditions even after four consecutive same-feature trials. Although RTs did not seem to diminish further from two to three repetitions, additional repetitions would be necessary to ensure that PoP effects on RTs reached an asymptotic level. However, the fact that the differences in distractor interference and search slopes did not diminish over three repetitions establishes the fact that PoP cannot account for the differences on these measures.

It is noteworthy that the effects of nontarget feature repetition were significant only in the mixed singleton condition, not in the fixed singleton condition, which points to an additional difference between these conditions. This finding, however, might be idiosyncratic to the procedure used in the present experiment. In the mixed singleton condition, the target on one trial sometimes became the nontarget on the next trial, or vice versa, whereas in the fixed singleton condition, the target and the nontarget features were never exchanged. Thus, negative priming effects might have inflated the effect of nontarget feature repetition in the mixed singleton condition, relative to the fixed singleton condition. ${ }^{7}$ In order to address this concern, we computed nontarget feature repetition effects in the mixed singleton condition (Experiment 3), while excluding trials that were potentially contaminated by negative priming effects. This analysis revealed a 29-msec facilitation on repeated nontarget feature trials, but although this effect was in the expected direction, it did not reach significance $[F(1,11)=2.95, p>.1]$. It would be important to further examine this issue because earlier reports of nontargetfeature repetition effects (Maljkovic \& Nakayama, 1994, Experiment 8) may have been contaminated by top-down expectancies. Indeed, in that study, the probability that the nontarget feature would be repeated on the next trial was considerably higher than the probability for a different nontarget feature trial. Thus, it is not clear at this point whether nontarget feature repetition in pop-out search indeed facilitates performance.

\section{GENERAL DISCUSSION}

The results of two experiments replicate the basic phenomena observed in earlier research on singleton search (e.g., Bacon \& Egeth, 1994; Bravo \& Nakayama, 1992; Maljkovic \& Nakayama, 1994; Theeuwes, 1992). However, the novel findings reported in the present study challenge the current interpretations of these phenomena. They call for a reassessment of the distinction between the singleton detection and the feature search modes and cast new light on the role of intertrial memory processes in visual search.

\section{Reassessment of the Notion of a Default Singleton Detection Mode}

The present results clearly do not support the notion of a default singleton detection mode that is blind to additional feature information or the claim that the same saliencebased mechanism underlies search for a singleton target whether its specific feature is known (fixed singleton) or unknown (mixed singleton) to the observer (e.g., Bacon \& Egeth, 1994; Maljkovic \& Nakayama, 1994; Theeuwes, 1992). Indeed, performance in the two types of search differed in several ways. Mean RT was higher and distractor interference was larger in the mixed singleton than in the fixed singleton condition. Moreover, the search slope was negative in the mixed singleton condition but flat in the fixed singleton condition.

In contrast to Maljkovic and Nakayama's (1994) suggestion, intertrial feature repetition effects did not eliminate these differences. They did not interact with either display size or distractor presence and did not, therefore, even reduce the differences between the mixed and the fixed singleton conditions on these measures. Moreover, although intertrial feature repetition effects substantially shortened mean RTs in the mixed singleton condition, these remained considerably longer than those in the fixed singleton condition (Experiment 2). Because we examined the effects of only up to 3 repetitions, the possibility remains that with more repetitions, the differences between the fixed and the mixed singleton conditions might disappear. This would imply that in addition to a short-term component (due to short-lasting contributions from individual trials) that does not affect search slopes and distractor interference, repetition effects might also have a long-term component (due to nonlinear effects from many repetitions) that does affect search slopes and 
distractor interference. ${ }^{8}$ This account of the present data seems unlikely for two reasons. First, the typical curve depicting PoP effects is a power function, with improvement in performance being large on the first repetitions and smaller as the number of repetitions increases (with up to 10 repetitions in Maljkovic \& Nakayama's [1994] study). Second, should one observe the hypothesized long-term repetition effects, it would seem reasonable to attribute such long-term effects to a change in strategy, rather than to repetition effects: With enough repetitions, subjects might be induced to expect the target feature to remain constant.

The absence of an interaction between distractor interference and target feature repetition appears to be directly at odds with recent findings by Pinto, Olivers, and Theeuwes (2005). Their study was similar to the present Experiment 1 but included only the fixed and mixed singleton conditions. They found that on repeated target feature trials, interference by an irrelevant distractor was markedly reduced, relative to switched feature trials in the mixed singleton condition, and of the same magnitude as the interference observed in the fixed singleton condition. Thus, in that study, PoP appeared to eliminate the difference in the magnitude of distractor interference between the fixed and the mixed singleton conditions.

The main difference between Pinto et al.'s (2005) study and ours is that the distractor-present and distractor-absent conditions were presented in separate blocks in their experiments, whereas in ours, they were intermixed within blocks of trials. It should be noted that using a blocked design entails that task demands are likely to differ between the blocked conditions. Our objective, as well as Pinto et al.'s, was to evaluate the role of top-down factors in search for a known singleton target, so that it was imperative that task demands be kept strictly identical in all the relevant conditions. Accordingly, because the subjects' attentional set may have been different in the distractor-present and the distractor-absent conditions, Pinto et al.'s results are not informative as to whether repeating the target feature reduces distractor effects. Instead, they are informative as to the modulation of PoP by task demands. Indeed, they showed that PoP effects were substantially larger in the distractorpresent block than in the distractor-absent block, which indicates that the attentional set induced by the presence of a distractor on each trial enhanced PoP effects.

On the basis of the claim that performance in the fixed and the mixed singleton conditions became similar with respect to distractor interference after one repetition of the target feature, Pinto et al. (2005) concluded that the same salience-based mechanism underlies the two conditions and that no feature-based guidance operates in search for a known singleton. However, in their study, RTs in the mixed singleton condition on repeated target feature trials remained substantially higher than those in the fixed singleton condition. Pinto et al. attributed this difference to postselective factors - namely, to the comparison of the selected item with a target template in visual short-term memory (Duncan \& Humphreys, 1989). Note that this account implies a very inefficient mechanism. On the one hand, search for a singleton is assumed to rely exclusively on a salience-based mechanism, with no role for top-down knowledge even when it uniquely specifies the target. But on the other hand, subjects are assumed to engage in a feature-based template-matching process after the target has been selected. In the absence of corroborating evidence, this postselective account remains speculative. To conclude, then, although the findings reported by Pinto et al. bring into light the role of task demands on PoP, they do not offer strong support for the idea that feature-based guidance plays no role in singleton search, because in their experiments, (1) differences in task demands between the distractor-present and the distractor-absent conditions did not allow one to assess the effects of PoP on distractor interference and (2) PoP effects did not account for all the RT difference between the fixed and the mixed singleton conditions.

In the present study, we observed considerable repetition effects, but these did not eliminate all the differences between the fixed and the mixed singleton conditions. Since stimulus-driven factors (displays) were identical in the three conditions, can we conclude that the remaining differences in performance should be attributed to strategic factors? A closer look at the data suggests that we can. Recently, Leber and Egeth $(2003,2006)$ reported strategic carryover effects from one block of trials to the next in singleton search. Specifically, in search for a known singleton (fixed singleton condition) where two different attentional sets could guide subjects to the target (knowledge of the target feature and knowledge that the target is a singleton), the subjects appeared to remain with the attentional set that they were forced to use in the preceding block (salience based vs. feature based). In the present study, the differences between the fixed and the mixed types of singleton search that were not modulated by intertrial repetition effects were distractor interference and search slopes. Finding that these effects are influenced by the search condition in the preceding block would support the claim that attentional set (or top-down factors) account for the differences between the fixed and the mixed singleton conditions that were not modulated by repetition effects. To examine this issue, we compared distractor presence and display size effects in the fixed singleton condition when this condition had immediately followed the mixed singleton condition versus the multiple target condition in Experiment 1. The interaction between previous block (mixed singleton vs. multiple target) and distractor presence approached significance $[F(1,15)=3.24, p<.1]$, with a tendency for target interference in the fixed singleton condition to be larger after the mixed singleton than after the multiple target condition (42 vs. $4 \mathrm{msec}$, respectively). The interaction between previous block and display size also approached significance $[F(1,15)=4.02$, $p<.07]$, with a tendency for steeper search slopes in the fixed singleton condition following the multiple target condition than following the mixed singleton condition ( 5.25 vs. $0.75 \mathrm{msec} /$ item, respectively). These data suggest that attentional set plays a role in singleton search. However, because the relevant effects only approached 
significance and resulted from post hoc comparisons, further research is needed to establish this role.

Taken together, the present findings are consistent with the idea that subjects take advantage of the two sources of knowledge available - that is, that they search for a discontinuity and for a specific feature. Accordingly, these findings can be interpreted as follows. (1) RTs were shortest in the fixed singleton condition because this condition benefited from two, rather than only one, source of guidance. (2) The magnitude of distractor interference was intermediate in the fixed singleton condition because the attentional weight allocated to singletons in the fixed singleton condition was smaller than that in the mixed singleton condition and larger than that in the multiple target condition. (3) Increasing the number of nontargets (i.e., display density) rendered search based on salience faster, whereas it slightly slowed search based on feature, so that search based on both types of knowledge exhibited virtually flat slopes.

In line with Bacon and Egeth's (1994) main thrust, the present study supports the notion that search can be guided by knowledge that the target is a singleton, thus arguing against the idea that salience affects search in a purely stimulus-driven fashion (e.g., Kim \& Cave, 1999; Theeuwes, Atchley, \& Kramer, 2000). However, we also found that this set can be used concomitantly with a set for a specific feature, which disqualifies it as a distinct search mode (singleton detection mode) that is mutually exclusive with a set for a specific feature (feature search mode).

\section{A Role for Stimulus-Driven Salience in Feature Search Mode}

The present results contradict the notion of a feature search mode that is impervious to stimulus-driven salience effects (e.g., Bacon \& Egeth, 1994; Folk \& Remington, 1998; Lamy \& Tsal, 1999; Yantis \& Egeth, 1999). Indeed, in the multiple target condition, distractor interference, although absent in low-density displays, emerged in highdensity displays, indicating that stimulus-driven salience affected performance (see Theeuwes, 2004, for a similar finding). It is also consistent with the notion of attentional misguidance by irrelevant singletons (Todd \& Kramer, 1994; Yantis \& Egeth, 1999, Experiment 3) and with the effects of salience on interference by a distractor singleton on the task-relevant dimension (Lamy et al., 2004). However, Bacon and Egeth (1994, Experiment 2) failed to observe such density effects with similar displays, display sizes, and task. It is not clear at this point what differences between our study and theirs might account for this discrepancy.

Finally, the present findings also argue against Theeuwes's (2004) alternative account of the absence of singleton distractor interference reported by Bacon and Egeth (1994, Experiments 2 and 3) in feature-guided search. Theeuwes (2004) suggested that subjects adjust the size of their attentional window according to task demands. When searching for the target is difficult (for instance, because of target-nontarget heterogeneity), search is partly serial, whereas when search is easy, the attentional window can encompass the whole visual field, and search is therefore parallel. He showed that with large display sizes (12 and 20), search for a nonsingleton shape (a diamond among one square, one triangle, and 9 or 17 circles) was parallel and significantly slowed by the presence of an irrelevant color singleton (Experiment 1). In contrast, the same search with smaller display sizes ( 2 and 6 circles, instead of 9 and 17) was slightly positive (about $12 \mathrm{msec} /$ item) and insensitive to the presence of an irrelevant singleton. However, this account cannot accommodate our data, since we reported an instance of parallel search (i.e., a search slope not significantly different from 0) with no distractor interference. Indeed, in Experiment 1, mean search slope in the multiple target condition was $6.5 \mathrm{msec}(F<1)$, and distractor interference was $9 \mathrm{msec}$ $(F<1$; see also Leber \& Egeth, 2003). In order to further test Theeuwes's (2004) idea that distractor interference in search for a nonsingleton target is contingent on flat search slopes, we also calculated the correlation between search slope and distractor interference in the multiple target condition in the present Experiment 1. The mean search slope and the interference score were calculated for each subject, and the correlation was calculated across subjects. This correlation should be negative if distractor effects diminish with more positive search slopes. The correlation approached significance $(r=.48, p<.06)$, but the relationship was in the direction opposite of that predicted by Theeuwes's (2004) account; that is, interference tended to be larger for subjects who displayed steeper search slopes.

\section{Intertrial Memory Processes in Visual Search}

The recent literature suggests that various intertrial memory processes play an important role in guiding visual search (e.g., contextual cuing, Chun \& Jiang, 1998; dimensional weighting, Müller, Heller, \& Ziegler, 1995; negative priming, DeSchepper \& Treisman, 1996; see Shore \& Klein, 2000, for a review). In this article, we have focused on one class of such intertrial memory processes: priming of pop-out (Goolsby \& Suzuki, 2001; Hillstrom, 2000; Hodsoll \& Humphreys, 2001; Maljkovic \& Nakayama, 1994, 1996, 2000; Wolfe et al., 2003). We replicated the basic PoP finding in both experiments.

Maljkovic and Nakayama (1994, 1996, 2000; McPeek, Maljkovic, \& Nakayama, 1999) attributed PoP effects to the operation of a short-term implicit memory system that speeds the deployment of attention and execution of saccadic eye movements to a repeated attention-driving feature. However, these authors did not specify the stage in attentional deployment that is affected by PoP. As has been noted by Wolfe et al. (2003), PoP cannot affect attentional priority ranking in pop-out search, because search slopes are typically null or negative, which indicates that attention is almost always deployed to the target first. In order to explain why search tasks with similar near-zero slopes have markedly different mean RTs (e.g., mixed vs. fixed singleton conditions in the present study), Wolfe et al. proposed that RT might be based on the salience of the difference between the target and the nontargets (signal-to-noise, or $\mathrm{S} / \mathrm{N}$, ratio), rather than being based 
simply on the rank order position of the target on a list of the salience or activation of items. In other words, even with $\mathrm{S} / \mathrm{N}$ ratios high enough to ensure that the target is the first item attended to, an increase in the $\mathrm{S} / \mathrm{N}$ ratio would result in shorter RTs. Within this conceptual framework, Wolfe et al. further suggested that PoP increases the signal emanating from a repeated feature and, thus, speeds mean RTs without affecting search slopes, which is precisely the pattern of results we observed.

According to this view, PoP should also reduce the effects of any factor that increases noise. However, this is not what we found: PoP did not reduce the very large distractor interference observed in the mixed singleton condition. This finding suggests that although the signal emanating from the irrelevant singleton was stronger than that emanating from the target (hence, the cost associated with the irrelevant singleton presence), this difference was not reduced when the target feature benefited from PoP. It follows that PoP may not affect the $\mathrm{S} / \mathrm{N}$ ratio ${ }^{9}$ but, perhaps, the speed at which attention can be moved to the target location, after this move has been programmed on the basis of attentional weights or the $\mathrm{S} / \mathrm{N}$ ratio.

\section{REFERENCES}

Bacon, W. F., \& Egeth, H. E. (1994). Overriding stimulus-driven attentional capture. Perception \& Psychophysics, 55, 485-496.

Bravo, M. J., \& NaKaYAMa, K. (1992). The role of attention in different visual-search tasks. Perception \& Psychophysics, 51, 465-472.

Chun, M. M., \& Jiang, Y. (1998). Contextual cueing: Implicit learning and memory of visual context guides spatial attention. Cognitive Psychology, 36, 28-71.

DeSchepper, B., \& Treisman, A. (1996). Visual memory for novel shapes: Implicit coding without attention. Journal of Experimental Psychology: Learning, Memory, \& Cognition, 22, 27-47.

Duncan, J., \& Humphreys, G. W. (1989). Visual search and stimulus similarity. Psychological Review, 96, 433-458.

Egeth, H. E., Jonides, J., \& Wall, S. (1972). Parallel processing of multielement displays. Cognitive Psychology, 3, 674-698.

Folk, C. L., Leber A. B., \& Egeth, H. E. (2002). Made you blink! Contingent attentional capture produces a spatial blink. Perception \& Psychophysics, 64, 741-753.

FolK, C. L., \& Remington, R. [W.] (1998). Selectivity in distraction by irrelevant featural singletons: Evidence for two forms of attentional capture. Journal of Experimental Psychology: Human Perception \& Performance, 24, 847-858.

Folk, C. L., Remington, R. W., \& Johnston, J. C. (1992). Involuntary covert orienting is contingent on attentional control settings. Journal of Experimental Psychology: Human Perception \& Performance, 18, 1030-1044.

Goolsby, B. A., \& SuzuKI, S. (2001). Understanding priming of colorsingleton search: Roles of attention at encoding and "retrieval." Perception \& Psychophysics, 63, 929-944.

Hillstrom, A. P. (2000). Repetition effects in visual search. Perception \& Psychophysics, 62, 800-817.

Hodsoll, J., \& Humphreys, G. W. (2001). Driving attention with the top down: The relative contribution of target templates to the linear separability effect in the size dimension. Perception \& Psychophysics, 63, 918-926.

Kim, M.-S., \& CAVE, K. R. (1999). Top-down and bottom-up attentional control: On the nature of interference from a salient distractor. Perception \& Psychophysics, 61, 1009-1023.

Lamy, D., Bar-Anan, Y., Egeth, H. E., \& Carmel, T. (2006). Effects of top-down guidance and singleton priming on visual search. Psychonomic Bulletin \& Review, 13, 287-293.

Lamy, D., \& Egeth, H. E. (2003). Attentional capture in singleton- detection and feature-search modes. Journal of Experimental Psychology: Human Perception \& Performance, 29, 1003-1020.

Lamy, D., Leber, A. B., \& Egeth, H. E. (2004). Effects of bottom-up salience within feature-search mode. Journal of Experimental Psychology: Human Perception \& Performance, 30, 1019-1031.

Lamy, D., \& Tsal, Y. (1999). A salient distractor does not disrupt conjunction search. Psychonomic Bulletin \& Review, 6, 93-98.

Lamy, D., Tsal, Y., \& Egeth, H. E. (2003). Does a salient distractor capture attention early in processing? Psychonomic Bulletin \& Review, 10, 621-629.

Leber, A. B., \& Egeth, H. E. (2003, November). It's under control: Top down search strategies do override attentional capture. Paper presented at the annual Object Perception, Attention, and Memory (OPAM) workshop, Vancouver.

Leber, A. B., \& Egeth, H. E. (2006). Attention on autopilot: Past experience and attentional set. Visual Cognition, 14, 565-583.

Maljkovic, V., \& Nakayama, K. (1994). Priming of pop-out: I. Role of features. Memory \& Cognition, 22, 657-672.

Maljkovic, V., \& NaKayama, K. (1996). Priming of pop-out: II. The role of position. Perception \& Psychophysics, 58, 977-991.

Maljkovic, V., \& Nakayama, K. (2000). Priming of popout: III. A short-term implicit memory system beneficial for rapid target selection. Visual Cognition, 7, 571-595.

McPeek, R. M., Maljkovic, V., \& Nakayama, K. (1999). Saccades require focal attention and are facilitated by a short-term memory system. Vision Research, 39, 1555-1566.

Müller, H. J., Heller, D., \& Ziegler, J. (1995). Visual search for singleton feature targets within and across feature dimensions. Perception \& Psychophysics, 57, 1-17.

PASHLER, H. (1988). Cross-dimensional interaction and texture segregation. Perception \& Psychophysics, 43, 307-318.

Pinto, Y., Olivers, C. N. L., \& Theeuwes, J. (2005). Target uncertainty does not lead to more distraction by singletons: Intertrial priming does. Perception \& Psychophysics, 67, 1354-1361.

RAUSCHENBERGER, R. (2003). Attentional capture by auto- and allocues. Psychonomic Bulletin \& Review, 10, 814-842.

RuZ, M., \& LupiáÑEZ, J. (2002). A review of attentional capture: On its automaticity and sensitivity to endogenous control. Psicológica, 23, 283-309.

Shore, D. I., \& Klein, R. M. (2000). On the manifestations of memory in visual search. Spatial Vision, 14, 59-75.

Theeuwes, J. (1992). Perceptual selectivity for color and form. Perception \& Psychophysics, 51, 599-606.

Theeuwes, J. (2004). Top-down search strategies cannot override attentional capture. Psychonomic Bulletin \& Review, 11, 65-70.

Theeuwes, J., Atchley, P., \& Kramer, A. F. (2000). On the time course of top-down and bottom-up control of visual attention. In S. Monsell \& J. Driver (Eds.), Control of cognitive processes: Attention and performance XVIII (pp. 105-124). Cambridge, MA: MIT Press.

TodD, S., \& Kramer, A. F. (1994). Attentional misguidance in visual search. Perception \& Psychophysics, 56, 198-210.

Wolfe, J. M., Butcher, S. J., Lee, C., \& Hyle, M. (2003). Changing your mind: On the contributions of top-down and bottom-up guidance in visual search for feature singletons. Journal of Experimental Psychology: Human Perception \& Performance, 29, 483-502.

Yantis, S., \& EgEth, H. E. (1999). On the distinction between visual salience and stimulus-driven attentional capture. Journal of Experimental Psychology: Human Perception \& Performance, 25, 661-676.

\section{NOTES}

1. In this article, a parallel search will be operationally defined as a search for which performance does not deteriorate as the number of nontargets increases.

2. It is important to note that the logical basis for this conclusion is contestable. Even if performance in search for a known singleton could be shown to be indistinguishable from performance in search for an unknown singleton after repetition effects are taken into account, it would not necessarily imply that the same mechanism underlies the two types of search. Such a finding might simply indicate that when top-down factors are not available to guide search, implicit memory processes (active 
in the mixed condition) yield facilitation effects of the same magnitude as top-down knowledge of target feature used in the blocked condition. In the present article, we will show that repetition effects alone cannot account for all the differences between the different search conditions we will examine. Accordingly, we will not have to come to grips with this criticism of Maljkovic and Nakayama's (1994) inferences.

3. Maljkovic and Nakayama (1994) rejected the possibility that expectancy of the upcoming color played any role in the difference between the blocked and the mixed conditions (Experiments 2 and 4). However, although their results indeed suggest that PoP played a more significant role than did expectancy, they do not allow one to conclude that expectancy played no role.

4. In both experiments, preliminary analyses showed no main effect of order of search condition for either the RT or the accuracy data, but the interaction between this factor and search condition was significant for the RT data. This effect is attributable to practice: Any given search condition was faster when it was presented at a later stage in the experiment. The data were, therefore, collapsed across search condition orders.

5. In this experiment, the effects of target feature repetition are confounded with the effects of nontarget feature repetition, because these features are randomly switched from trial to trial. For the sake of simplicity, however, these effects will be referred to as target feature repetition or PoP effects.

6. Additional differences were observed on target-absent trials of a target detection task from an unpublished experiment. This experiment was identical to the present Experiment 1, except that the subjects had to detect the presence of the target, rather than to categorize the orientation of the line presented inside of it. On target-absent trials, in which PoP could play no role, RTs were much higher in the mixed than in the fixed singleton condition. Moreover, whereas search was faster on target-present than on target-absent trials in the fixed singleton and multiple target conditions, the reverse was true in the mixed singleton condition. The pattern of performance on target-present trials was qualitatively identical to that obtained with the discrimination task used in Experiment 1-namely, significant PoP effects were observed, but they did not close the gap between the fixed and the mixed singleton conditions in terms of overall RTs and distractor interference (search slopes were null). Although these findings are consistent with the conclusions from Experiment 1, it may be important to keep in mind that the role of PoP might be different in detection tasks and in discrimination tasks. Indeed, the latter tasks require that attention be focused on the target, whereas in the former tasks, subjects may remain in a state of distributed attention and simply judge whether or not a discontinuity is present in the display. (We thank an anonymous reviewer for this suggestion.)

7. Note that such an alternative account based on negative priming may also apply to target feature repetition effects (Experiment 2) and overall PoP effects (Experiment 1). We are currently investigating this issue.

8 . We thank an anonymous reviewer for this suggestion.

9. Hillstrom (2000) arrived at a similar conclusion on the basis of the finding that target feature repetition did not reduce the positive slopes generated by a conjunction search. However, in her study, subjects were required to search for one of two possible targets presented in a totally predictable sequence. This task is fundamentally different from the mixed singleton condition in which there is only one target definition, with different individual targets matching this definition. It is thus reasonable to suggest that the target repetition effects observed by Hillstrom might reflect task-switching costs, rather than PoP.

(Manuscript received June 30, 2004; revision accepted for publication September 15, 2005.) 